\title{
RADIO-CONTINUUM STUDY OF MCSNR J0536-7038 (DEM L249)
}

\author{
L. M. Bozzetto ${ }^{1}$, M. D. Filipović ${ }^{1}$
}

\begin{abstract}
We present a detailed radio-continuum study on Australia Telescope Compact Array (ATCA) observations of Large Magellanic Cloud (LMC) supernova remnant (SNR), MCSNR J0536-7038. This Type Ia SNR follows a horseshoe morphology, with a size $32 \mathrm{pc}$ $\times 32 \mathrm{pc}$ (1-pc uncertainty in each direction). It exhibits a radio spectrum $\alpha=-0.52 \pm 0.07$ between $\lambda=73$ and $6 \mathrm{~cm}$. We report detections of regions showing moderately high fractional polarisation at $6 \mathrm{~cm}$, with a peak value of $71 \pm 25 \%$ and a mean fractional polarisation of $35 \pm 8 \%$. We also estimate an average rotation measure across the remnant of $-237 \mathrm{rad} \mathrm{m}^{-2}$. The intrinsic magnetic field appears to be uniformly distributed, extending in the direction of the two brightened limbs of the remnant.
\end{abstract}

\section{Introduction}

Supernova and their remnants play a crucial role in the evolution and structure of the interstellar medium (ISM), as they are responsible for the creation and distribution of the heavier elements and the chemical enrichment of galaxies. The Large Magellanic Cloud (LMC) is a favourable environment for observing supernova remnants (SNRs), due to the relatively close proximity in which it is located (and in turn larger angular size), making it possible to resolve these objects $\left(\right.$ as $\left.1 \mathrm{pc}=4^{\prime \prime}\right)$. One LMC SNR of particular interest is MCSNR J0536-7038, a Type Ia SNR that has prominent $\mathrm{Fe}$ emission in its core and is thought to be the result of a 'prompt' Type Ia event (Borkowski. Hendrick, \& Revnolds 2006; Maggi et al. 2014).

L. M. Bozzetto, M. D. Filipović

${ }^{1}$ University of Western Sydney, Locked Bag 1797, Penrith South DC, NSW 1797, Australia
MCSNR J0536-7038 was originally observed by Davies et al. (1976) using the 48-in SRC Schimdt camera, who gave this object the association DEM L249. They estimate an optical diameter of $180^{\prime \prime} \times 120^{\prime \prime}$ making note that this object was very faint and had diffused emission. Mathewson et al. (1983) find an optical size of $162^{\prime \prime} \times 129^{\prime \prime}$ and an X-ray size of $\sim 120^{\prime \prime}$, an integrated flux density at $408 \mathrm{MHz}$ of $130 \mathrm{mJy}$ and a radio spectral index of $\alpha=-0.53$. Mills et al. (1984) recorded an integrated flux density measurement at $843 \mathrm{MHz}$ of $850 \mathrm{mJy}$, updating the spectral index to $\alpha=-0.52$. They also note a horseshoe morphology for which they state that approximately one third of SNRs greater than $30^{\prime \prime}$ are. Fusco \& Preite (1984) find an ambient density of $0.05 \mathrm{~cm}^{-3}$, a shock temperature of $1.5 \mathrm{keV}$, an age of $6.5 \times 10^{3}$ years, total swept up mass of $55 M_{\odot}$ and a shock velocity of $1100 \mathrm{~km} \mathrm{~s}^{-1}$. No pulsar was found at this position by Manchester. Damico, \& Tuohv (1985) in their search for short period pulsars. Berkhuijsen (1986) measure a radio surface brightness of $20.5 \mathrm{~W} \mathrm{~Hz} \mathrm{~Hz}^{-1} \mathrm{sr}^{-1}$ using the radio diameter of $30 \mathrm{pc}$ and spectral index of $\alpha=-0.52$. Also measured was a X-ray surface brightness of $32.4 \mathrm{erg} \mathrm{s}^{-1} \mathrm{pc}^{-2}$ using the X-ray extent of 39 pc. Chu \& Kennicutt (1988) listed the OB association of $560 \mathrm{pc}$ to LH66,69 and listed the source as population type II? (with '?' indicating uncertain classification). They also made note that there was no detectable $\mathrm{CO}$ in the vicinity. Filipović et al. (1995) used the Parkes radio telescope and measured integrated flux densities of $25 \mathrm{mJy}(4750 \mathrm{MHz})$ and $34 \mathrm{mJy}(4850 \mathrm{MHz})$. Williams et al. (1999) listed an average brightness of $0.0010 \mathrm{cts} \mathrm{s}^{-1}$ arc $\mathrm{min}^{-2}$, an X-ray size of $5.2^{\prime} \times 3.3^{\prime}$ and stated that the category of this SNR was unclassified as it did not show an unambiguous detection in the ROSAT observations. Haberl \& Pietsch (1999) recorded an extent of 29.7" and gave this SNR the association HP[99] 1173. 
Urošević et al. (2005) included MCSNR J0536-7038 in their study of the $\Sigma$ - $D$ relationship. Using a diameter of $39 \mathrm{pc}$, an integrated flux density of $62 \mathrm{mJy}$ at $1400 \mathrm{MHz}$ (which was extrapolated from the values listed for $\alpha$ and $\mathrm{S}_{36}$ by Filipović et al. (1998)) and a spectrum of $\alpha=-0.61$, they calculated the surface brightness of this SNR to be $2.0 \times 10^{-21} \mathrm{~W} / \mathrm{M}^{2} \mathrm{~Hz}$ sr. Blair et al. (2006) record this SNR as a large faint optical shell SNR which is brighter on the eastern side of the shell. They make note that it may be a mixed morphology class, and that $\mathrm{C}_{I I I}$ is present in its spectra which may indicate that the SNR is on the near side of the LMC. Seok et al. (2008) estimated an integrated flux of 9 mJy $(4800 \mathrm{MHz})$. An optical extent of $3.1^{\prime} \times 2.3^{\prime}$ was recorded by using the MCSNR Atlas as published by Williams (http://www.astro.uiuc.edu/projects/atlas/). Payne et al. (2008) spectroscopically confirmed nature of this SNR based on the $[\mathrm{S} \mathrm{II}] / \mathrm{H} \alpha$ ratio. Desai et al. (2010) recorded an extent of $3.0^{\prime} \times 2.0^{\prime}$ and no detection of a YSO for this object nor association with the molecular clouds.

Here, we report on new radio-continuum observations of MCSNR J0536-7038. The observations, data reduction and imaging techniques are described in Section 2. The astrophysical interpretation of newly obtained moderate-resolution total intensity and polarimetric images in combination with the existing Magellanic Cloud Emission Line Survey (MCELS) images are discussed in Section 3.

\section{Observations and reduction}

We observed MCSNR J0536-7038 along with various other LMC SNRs via the ATCA on the $15^{\text {th }}$ and $16^{\text {th }}$ of November 2011, using the new Compact Array Broadband Backend (CABB) at array configuration EW367 and at wavelengths of 3 and $6 \mathrm{~cm}(\nu=9000$ and $5500 \mathrm{MHz}$ ). Baselines formed with the $6^{\text {th }}$ ATCA antenna were excluded, as the other five antennas were arranged in a compact configuration. The observations were carried out in the so called "snap-shot" mode, totaling 50 minutes of integration over a 14 hour period. PKS B1934-638 was used for flux density calibration 1 and PKS B0530-727 was used for secondary (phase) calibration. The phase calibrator was observed twice every hour for a total 78 minutes over the whole observing session. The MIRIAD2 2 (Sault. Teuben. \& Wright 1995) and KARMA 3 (Gooch 1995) software packages were used

\footnotetext{
${ }^{1}$ Flux densities were assumed to be $5.098 \mathrm{Jy}$ at $6 \mathrm{~cm}$ and 2.736 at $3 \mathrm{~cm}$.

${ }^{2}$ http://www.atnf.csiro.au/computing/software/miriad/

${ }^{3} \mathrm{http}: / /$ www.atnf.csiro.au/computing/software/karma/
}

for reduction and analysis. More information on the observing procedure and other sources observed in this project can be found in Bozzetto et al. (2012a, b., d, 2013) and De Horta et al. (2012).

The CABB $2 \mathrm{GHz}$ bandwidth is a 16 times improvement from the previous $128 \mathrm{MHz}$, and with the new higher data sampling has increased the sensitivity of the ATCA by a factor of 4 . The $2 \mathrm{GHz}$ bandwidth not only aids in high sensitivity observations, but also allows data to be split into channels which can then be used for measuring Faraday rotation across the entire bandwidth, at frequencies close enough that the $n \times 180^{\circ}$ ambiguities prevalent when making an estimate between distant frequencies, are no longer an issue.

Images were formed using MIRIAD multi-frequency synthesis (Sault \& Wieringa 1994) and natural weighting. They were deconvolved with primary beam correction applied. The same procedure was used for both $U$ and $Q$ stokes parameter maps.

The $3 \mathrm{~cm}$ image (Fig. 1) has a resolution (full width half maximum $(\mathrm{FWHM}))$ of $22.4^{\prime \prime} \times 15.7^{\prime \prime}\left(\mathrm{PA}=44.5^{\circ}\right)$ and an r.m.s noise of $0.1 \mathrm{mJy} /$ beam. Similarly, we made an image of MCSNR J0536-7038 at $6 \mathrm{~cm}$ (seen as contours in Fig. 1) which has a FWHM of $38.5^{\prime \prime} \times 24.2^{\prime \prime}\left(\mathrm{PA}=48.1^{\circ}\right)$ and an estimated r.m.s. noise of $0.2 \mathrm{mJy} /$ beam.

In addition to using our own observations, we measure the integrated flux density at several frequencies between $\lambda=36 \mathrm{~cm}$ and $6 \mathrm{~cm}$, and record these measurements along with previous measurements of this SNR in Table. 1 The $36 \mathrm{~cm}(843 \mathrm{MHz})$ measurements come from the Molonglo Synthesis Telescope (MOST; as described in Mills et al. 1984) and Sydney University Molonglo Sky Survey (SUMMS; Mauch et al. 2008) mosaic images. These $36 \mathrm{~cm}$ surveys are from two different sets of observations and we point that the MOST data comes with somewhat better $u v$ coverage and sensitivity. This would be the most likely reason for a difference in flux density estimates (14 mJy or $\sim 20 \%)$ at this wavelength. The $20 \mathrm{~cm}(1384 \mathrm{MHz})$ measurement is from the mosaic image described in Hughes et al. (2007), while the $6 \mathrm{~cm}(4800 \mathrm{MHz})$ measurement comes from a mosaic image published by Dickel et al. (2010). Errors in these measurements predominately arose from defining the edge of the remnant. However, we estimate that the error in these measurements are in the order of $10 \%$. Using these values from Table 1. we estimate a spectral index for MCSNR J0536-7038 of $\alpha=-0.52 \pm 0.07$ (Fig. 2). We note the gap in flux density between our $6 \mathrm{~cm}(5500 \mathrm{MHz})$ measurement and the measurement from the $6 \mathrm{~cm}(4800 \mathrm{MHz})$ Dickel et al. (2010) mosaic. This can be explained by 

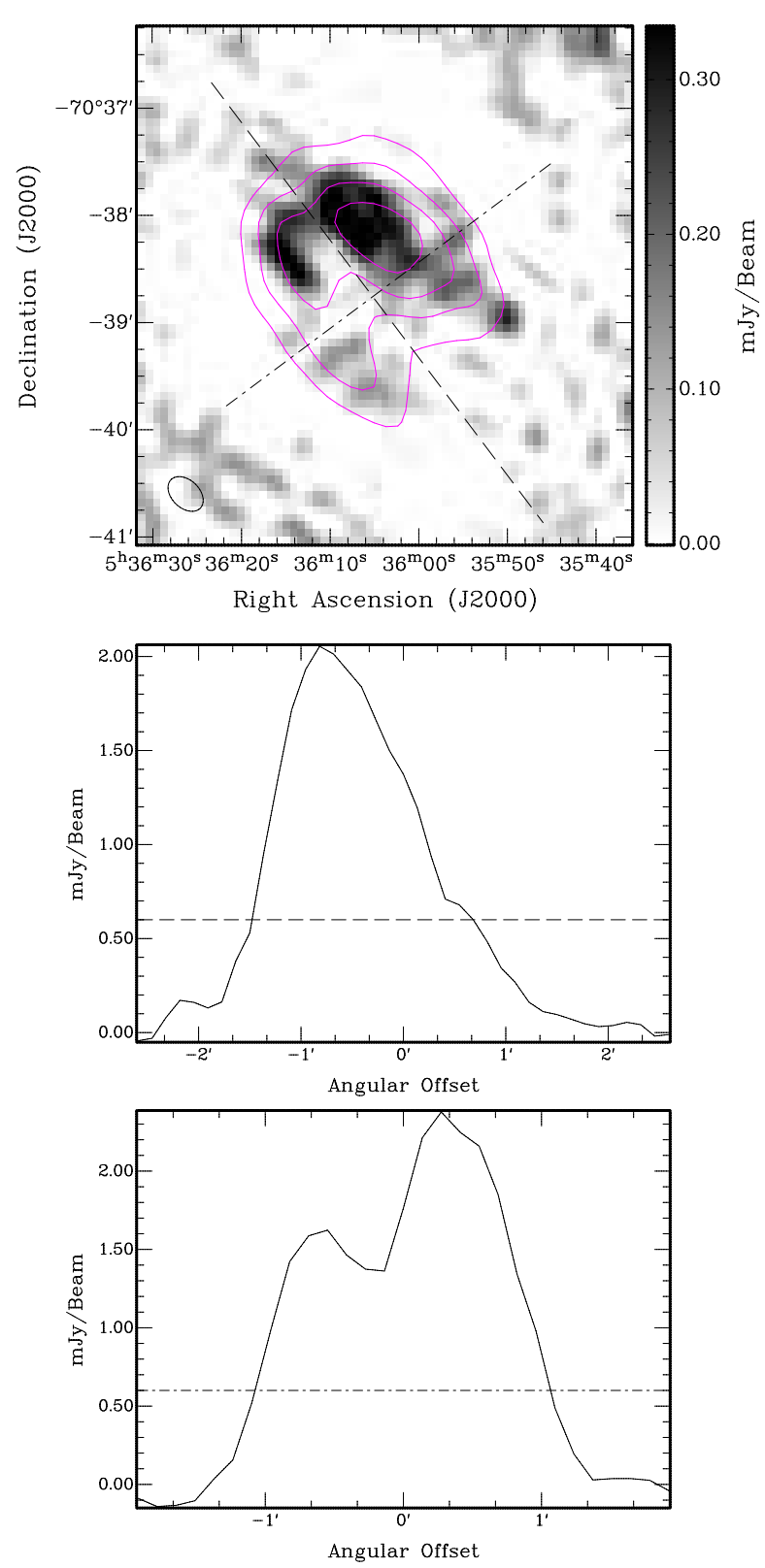

Fig. 1 The top image shows the $3 \mathrm{~cm}$ intensity image of MCSNR J0536-7038 overlaid with $6 \mathrm{~cm}$ contours. The contours are $3,6,9 \& 12 \sigma(\sigma=0.2 \mathrm{mJy} /$ beam $)$. The ellipse in the lower left corner represents the synthesised beamwidth at $3 \mathrm{~cm}$ of $22.4^{\prime \prime} \times 15.7^{\prime \prime}$. Also superimposed, are the major (NE-SW) and minor (SE-NW) axis. The middle and lower images show the intensity profile of the major and minor axis respectively, with an overlaid line at $3 \sigma$

the missing short (zero) spacing measurement in interferometery, which is responsible for the large scale, extended emission. The shortest gap in our baseline array of $46 \mathrm{~m}$ affected the amount of flux observed, whereas the Dickel et al. (2010) mosaic incorporated a single dish (and therefore, zero-spacing) measurement from the Parkes radio telescope in addition to ATCA observations. Flux density measurements taken from our $3 \mathrm{~cm}$ image were omitted from the spectral index calculation as the effects from short spacing were far more detrimental than at $6 \mathrm{~cm}$, as seen in Fig. 11. Measurements taken from the $3 \mathrm{~cm}$ Dickel et al. (2010) mosaic were also omitted as there was no reliable emission at the location of the remnant.

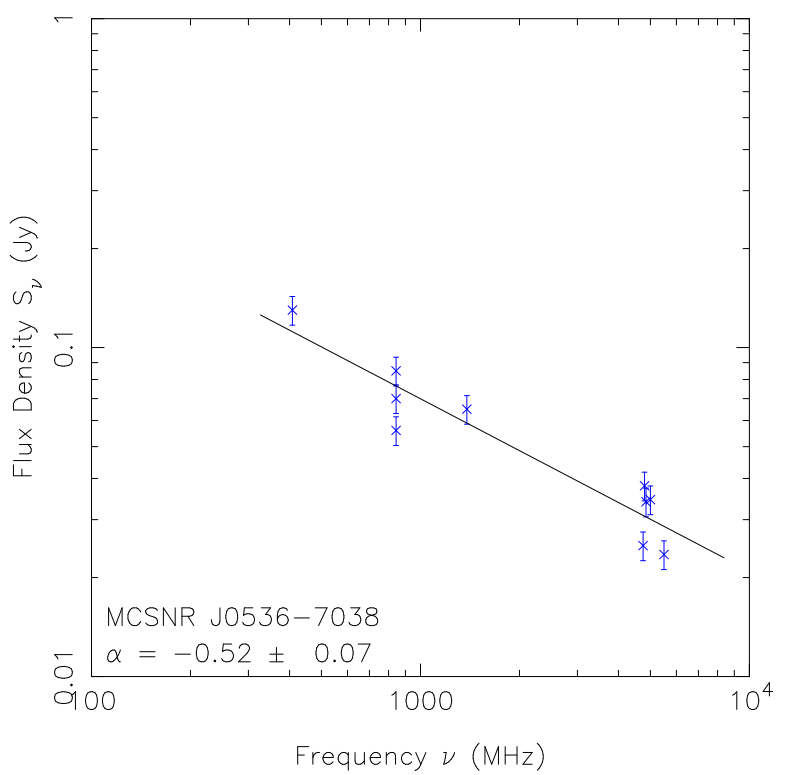

Fig. 2 Radio spectrum of MCSNR J0536-7038

We also used the Magellanic Cloud Emission Line Survey (MCELS) that was carried out with the $0.6 \mathrm{~m}$ University of Michigan/CTIO Curtis Schmidt telescope, equipped with a SITE $2048 \times 2048 \mathrm{CCD}$, which gave a field of $1.35^{\circ}$ at a scale of $2.4^{\prime \prime}$ pixel $^{-1}$. Both the LMC and SMC were mapped in narrow bands corresponding to $\mathrm{H} \alpha$, [O III] $(\lambda=5007 \AA)$, and [S II] $(\lambda=6716,6731 \AA)$. All the data has been flux-calibrated and assembled into mosaic images, a small section of which is shown in Fig. 3. Further details regarding the MCELS are given by Pellegrini et al. (2012) and at http://www.ctio.noao.edu/mcels.

\section{Results and Discussion}

MCSNR J0536-7038 exhibits a horseshoe morphology at $6 \mathrm{~cm}$ (Fig. 1) centered at RA (J2000) $=5^{h} 36^{\mathrm{m}}$ $07^{s} .7$, Dec $(\mathrm{J} 2000)=-70^{\circ} 38^{\prime} 20^{\prime \prime}$. We selected a onedimensional intensity profile across the approximate major (NE-SW) and minor (SE-NW) axes (Fig. 1) at the $3 \sigma$ noise level $(0.6 \mathrm{mJy})$ to estimate the spatial extent of the remnant. Its size at $6 \mathrm{~cm}$ is $130^{\prime \prime} \times 130^{\prime \prime}$ with a $4^{\prime \prime}$ uncertainty in each direction $(32 \times 32$ pc with a 1 pc uncertainty in each direction at the LMC distance of 
Table 1 Integrated flux density measurements for MCSNR J0536-7038.

\begin{tabular}{ccccccl}
\hline $\begin{array}{c}\nu \\
(\mathrm{MHz})\end{array}$ & $\begin{array}{c}\lambda \\
(\mathrm{cm})\end{array}$ & $\begin{array}{c}\text { r.m.s. } \\
(\mathrm{mJy} / \mathrm{beam})\end{array}$ & $\begin{array}{c}\text { Beam Size } \\
\left({ }^{\prime \prime}\right)\end{array}$ & $\begin{array}{c}\mathrm{S}_{\text {Total }} \\
(\mathrm{mJy})\end{array}$ & $\begin{array}{c}\Delta \mathrm{S}_{\text {Total }} \\
(\mathrm{mJy})\end{array}$ & Reference \\
\hline 408 & 73 & 40 & $157.2 \times 171.6$ & 130 & 13 & Mathewson et al. $(1983)$ \\
843 & 36 & - & $46.4 \times 43.0$ & 85 & 9 & Mills et al. (1984) \\
$843^{a}$ & 36 & 0.8 & $46.4 \times 43.0$ & 70 & 7 & This work \\
$843^{b}$ & 36 & 1.0 & $47.3 \times 45.0$ & 56 & 6 & This work \\
1384 & 20 & 0.6 & 40 & 65 & 7 & This work \\
4750 & 6 & 8 & 288 & 25 & 3 & Filipović et al. $(1995)$ \\
4800 & 6 & - & $35.0 \times 35.0$ & 38 & 4 & This work \\
4850 & 6 & 5 & 294 & 34 & 3 & Filipovic et al. $(1995)$ \\
5000 & 6 & - & 258 & 34.5 & 3 & Mathewson et al. $(1983)^{c}$ \\
5500 & 6 & 0.2 & $38.5 \times 24.2$ & 24 & 2 & This work \\
\hline
\end{tabular}

${ }^{a}$ Uses the MOST mosaic image.

${ }^{b}$ Uses the SUMMS mosaic image.

${ }^{c}$ This value was derived from the $408 \mathrm{MHz}$ flux value from Mathewson et al. (1983) and their spectral index.

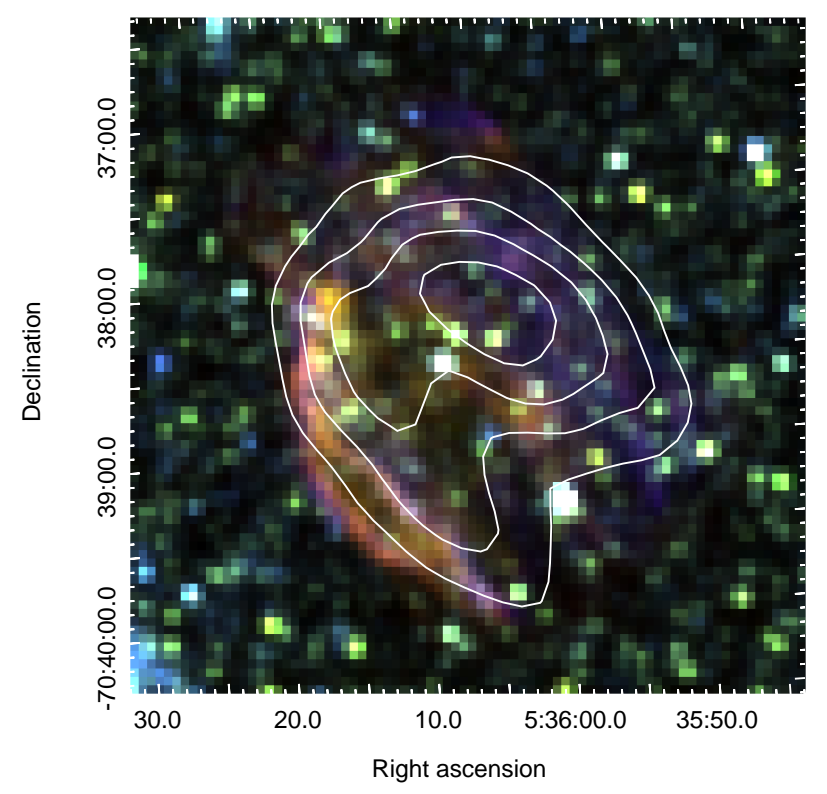

Fig. 3 MCELS composite optical image (RGB $=\mathrm{H} \alpha,[\mathrm{SiI}],[\mathrm{OIII}])$ of MCSNR J0536-7038 overlaid with $6 \mathrm{~cm}$ contours. The contours are $3,6,9$ and $12 \sigma(\sigma=$ $0.2 \mathrm{mJy} /$ beam)

$50 \mathrm{kpc}$ (di Benedetto 2008)). The remnant appears to be split into two limbs, the thicker, brighter limb toward the north-west and the thinner fainter limb towards the south-east.

The optical emission from the MCELS correlates nicely with our $6 \mathrm{~cm}$ radio-continuum emission. The [S II] and $\mathrm{H} \alpha$ emission are quite similar, with rather uniform emission spread over the remnant but ex- hibiting brightening on the south-east limb. [O III] emission is also found on this south-eastern limb (although thinner), however, unlike the $\mathrm{H} \alpha$ it is also found to show brightening towards the north-western limb. This is comparable to another LMC SNR that shares many similar properties (potential prompt Type Ia, older, opposite H $\alpha$ /[S II] - [O III]), MCSNR J0508-6902 (Bozzetto et al. 2014). The emission located towards the south-east of the remnant is a cluster of stars NGC 2056 and not likely associated with this SNR.

There has already been an extensive X-ray study done for this SNR. However, we have overlaid $6 \mathrm{~cm}$ radio contours on a three colour composite Chandra image (Obs. ID: 3908) to show the association between the X-ray and radio-continuum emission (Fig. 4). We find that the radio-continuum emission loosely follows the low energy X-ray band (0.45-1.03 keV) and the medium band $\mathrm{X}$-ray emission is confined in the interior of the remnant.

The spectral energy distribution (SED) of the remnant between $36 \mathrm{~cm}$ and $6 \mathrm{~cm}(\alpha=-0.52 \pm 0.07$; based on the values in Table 10 shows the nonthermal nature of this remnant at radio-wavelengths. This value of -0.5 is typical spectral index of SNRs (Mathewson et al. 1983; Filipović et al. 1998).

As we are unable to create a reliable $3 \mathrm{~cm}$ polarisation image (due to this wavelength being significantly affected by missing short spacing), we instead make use of the wide $2 \mathrm{GHz}$ bandwidth from the $6 \mathrm{~cm}$ observations in order to carry out a comparative polarisation study. The 2048 channels at $6 \mathrm{~cm}$ were split into two even parts, each consisting of 1024 channels. Both images were convolved to the same resolution so that they could be compared. 


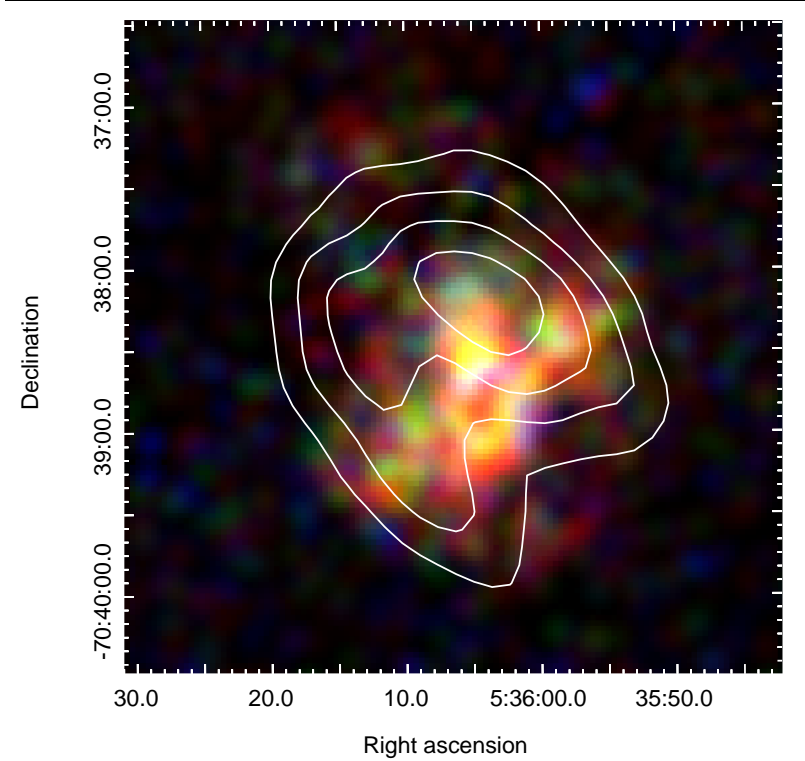

Fig. 4 Chandra three colour composite (red: 0.45$1.03 \mathrm{keV}$, green: $1.03-1.32 \mathrm{keV}$, blue: $1.32-2.2 \mathrm{keV}$ ) of MCSNR J0536-7038 overlaid with $6 \mathrm{~cm}$ radio contours of $369 \& 12 \sigma(\sigma=0.2 \mathrm{mJy})$

Fractional polarisation $(P)$ was calculated for both $6 \mathrm{~cm}$ images using:

$$
P=\frac{\sqrt{S_{Q}^{2}+S_{U}^{2}}}{S_{I}}
$$

where $S_{Q}, S_{U}$ and $S_{I}$ are integrated intensities for $Q, U$ and $I$ Stokes parameters. We estimate a mean fractional polarisation value of $P \cong 35 \pm 8 \%$ at $6 \mathrm{~cm}$. This relatively high level of polarisation is (theoretically) expected for an SNR with a radio spectrum of less than -0.5 (Rolfs \& Wilson 2003), which is in agreement with our spectral index of $\alpha \cong-0.52$. The structure of this polarisation can be seen in the top image of Fig. 5. where the electric field vectors at $6 \mathrm{~cm}$ have been superimposed on the $6 \mathrm{~cm}$ contours. These vectors are primarily facing the north-south direction, with slight change over the remnant, mostly found towards the norths and south limbs. We find a peak fractional polarisation for this SNR of $P=71 \pm 25 \%$. This level of polarisation is comparatively higher than most LMC SNRs, and is on par with the peak value found for LMC SNR J0455-6838 by Crawford et al. (2008), of $\sim 70 \%$.

High levels of intrinsic fractional polarisation (with a maximum of $p=(3-3 \alpha) /(5-3 \alpha)=\sim 70 \%$ in a ordered field) from synchrotron emission is possible, and we do expect the magnetic field components perpendicular to compression to be amplified. However, such levels of polarisation are not expected to be observed due to the non-uniform regions of the po- larised emission in addition to instrumental depolarisation and/or physical depolarisation effects outside the SNR. A possible explanation for these high values is given in Dickel, Milne, \& Strom (2000) where they pointed that the fractional polarisation can be artificially increased due to the polarised intensity having more fine scale structure than the total intensity, and therefore, the missing short spacing between antennas misrepresents the background level.

Polarisation position angles were taken from both $6 \mathrm{~cm}$ polarisation images and used to estimate Faraday rotation for this SNR. The rotation measure for this remnant can be seen in Fig. 5, where positive rotation measure is shown by filled boxes and negative rotation by empty boxes. The rotation measure is predominately positive across the remnant, with a few negative values found towards the edge of the remnant. However, the polarised intensity where these negative values are situated is too low to accurately determine rotation measure, and are therefore, probably not real. The average rotation measure across the remnant is $237 \mathrm{rad} \mathrm{m}^{-2}$.

To see the intrinsic magnetic field of MCSNR J0536-7038, we first de-rotate the $6 \mathrm{~cm}$ electric field vectors to their zero-wavelength position angle, and then rotate the vectors by 90 degrees to get the perpendicular magnetic field. The result of this can be seen in Fig. 5, where we find the magnetic field vectors overlaid on $6 \mathrm{~cm}$ contours. The vectors appear uniform across the field, with slight deviations along the east side and a couple on the northern most line. However, all lie within the $10-20 \%$ of $6-9 \sigma$ which makes them $1-2 \sigma$, and therefore not significant. The overall morphology suggests that MCSNR J0536-7038 went off in a region of uniformly NE-SW field and so compressed it more in the NW-SE direction.

From the position of MCSNR J0536-7038 at the surface brightness to diameter $(\Sigma-\mathrm{D})$ diagram ((D, $\left.\Sigma)=\left(32 \mathrm{pc}, 3.6 \times 10^{-21} \mathrm{~W} \mathrm{~m} \mathrm{mz}^{-1} \mathrm{sr}^{-1}\right)\right)$ by Berezhko \& Völk (2004), we can estimate that the remnant is likely to be an SNR in the late energy conserving phase, with an explosion energy between 0.25 and $1 \times$ $10^{51}$ ergs, which evolves in an environment of density $\sim 1 \mathrm{~cm}^{-3}$.

\section{Conclusions}

We provide a radio-continuum study of MCSNR J0536-7038, measuring an extent of $32 \mathrm{pc} \times 32 \mathrm{pc}$, strong polarisation across the remnant, with a mean of $35 \%$, nice optical to radio association for the SNR. We also find the intrinsic magnetic field for this remnant, which uniformly follows the path of the compressed emission. We 
estimate a spectrum of $\alpha=-0.52$ between $\lambda=6 \mathrm{~cm} \&$ $36 \mathrm{~cm}$, typical of a mid to older remnant. The rotation measure across the remnant is predominately positive with a mean rotation measure of $-237 \mathrm{rad} \mathrm{m}^{-2}$.

\section{Acknowledgements}

The Australia Telescope Compact Array is part of the Australia Telescope which is funded by the Commonwealth of Australia for operation as a National Facility managed by CSIRO. The MCELS is funded through the support of the Dean B. McLaughlin fund at the University of Michigan and through NSF grant 9540747. The scientific results reported in this article are based on observations made by the Chandra X-ray Observatory (CXO). We thank the referee for numerous helpful comments that have greatly improved the quality of this paper.
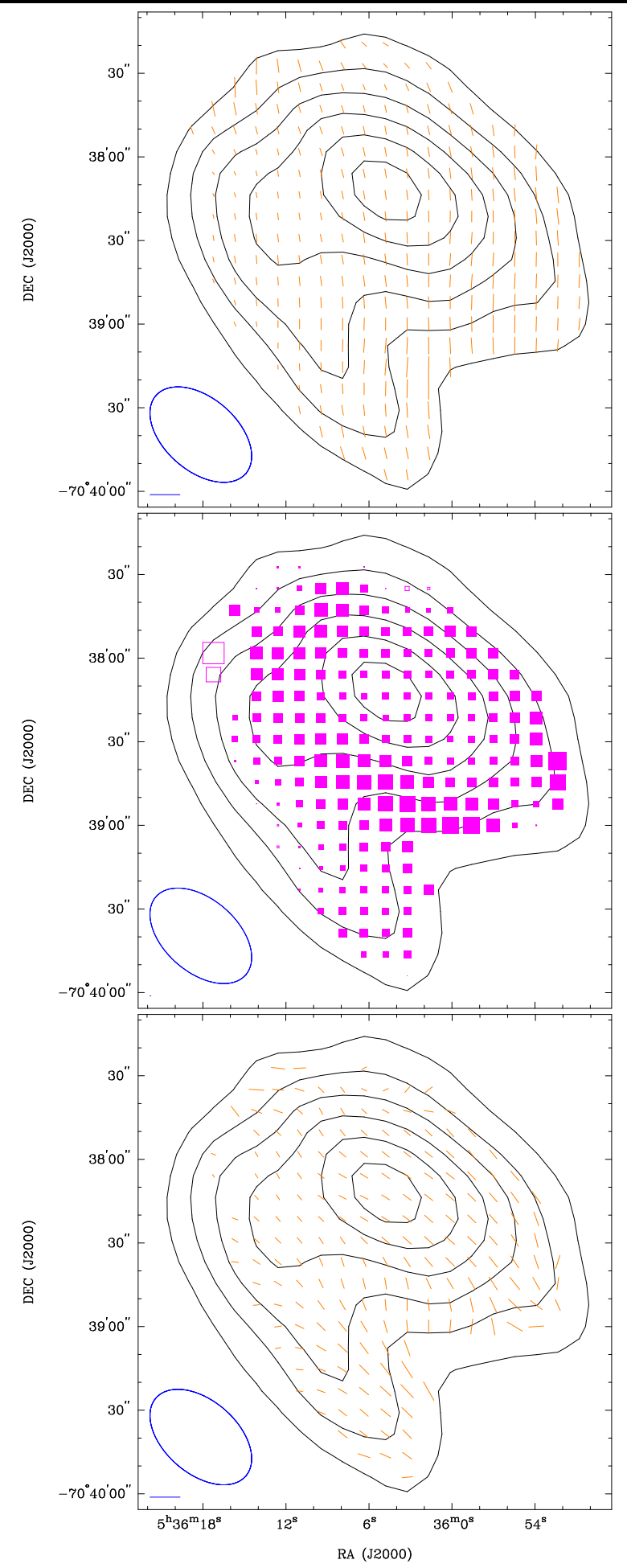

Fig. 5 Top - electric field of MCSNR J0536-7038 at $6 \mathrm{~cm}$. Middle - rotation measure, with filled boxes representing positive rotation and open boxes representing negative rotation. Bottom - Intrinsic magnetic field vectors with the polarised intensity based on $6 \mathrm{~cm}$ values. All images are overlaid with $6 \mathrm{~cm}$ contours of $3,6,9,12,15 \& 18 \sigma(\sigma$ $=2 \mathrm{mJy} /$ beam $)$. The ellipse in the lower left corner represents the synthesised beamwidth of $42.7^{\prime \prime} \times 26.1^{\prime \prime}$ and the line directly below (in the top and bottom images) shows a polarisation vector of $100 \%$ 


\section{References}

Berezhko E. G., Völk H. J., 2004, A\&A, 427, 525

Berkhuijsen, E. M., 1986, A\&A, 166, 257

Blair, W. P., Ghavamian, P., Sankrit, R. and Danforth, C. W., 2006, ApJS, 165, 480

Borkowski, K. J., Hendrick, S. P., Reynolds, S. P. 2006, ApJ, 652, 1259

Bozzetto L. M. et al., 2012a, MNRAS, 420, 2588

Bozzetto L. M., Filipović M. D., Crawford E. J., Payne J. L., de Horta A. Y., Stupar M. 2012b, Rev. Mex. Astron. Astros., 48, 41

Bozzetto, L. M., Filipović, M. D., Crawford, E. J., De Horta, A. Y. and Stupar, M., 2012c, Serbian Astronomical Journal, 184, 69

Bozzetto, L. M., Filipović, M. D., Urosevic, D. and Crawford, E. J., 2012d, Serbian Astronomical Journal, 185, 25

Bozzetto L. M., et al., 2013, MNRAS, 432, 2177

Bozzetto L. M., et al., 2014, arXiv:1401.1868

Chu, Y.-H. and Kennicutt, Jr., R. C., 1988, AJ, 96, 1874

Crawford, E. J., Filipović, M. D., de Horta, A. Y., Stootman, F. H., Payne, J. L. 2008, SAJ, 177, 61

Davies, R. D., Elliott, K. H. and Meaburn, J., 1976, MmRAS, 81, 89

De horta, A. Y., et al., 2012, A\&A, 540, A25

Desai, K. M., Chu, Y.-H., Gruendl, R. A., Dluger, W., Katz, M., Wong, T., Chen, C.-H. R., Looney, L. W., Hughes, A., Muller, E., Ott, J. and Pineda, J. L., 2010, AJ, 140, 584

Dickel, J. R., Milne, D. K. and Strom, R. G., 2000, ApJ, 543,840

Dickel J. R., McIntyre V. J., Gruendl R. A., Milne D. K., 2010, AJ, 140, 1567

di Benedetto G. P., 2008, MNRAS, 390, 1762

Filipović, M. D., Haynes, R. F., White, G. L., Jones, P. A., Klein, U., Wielebinski, R. 1995, A\&AS, 111, 311

Filipović, M. D., Haynes, R. F., White, G. L., Jones, P. A., 1998, A\&AS, 130, 421

Fusco-Femiano, R. and Preite-Martinez, A., 1984, ApJ, 281, 593

Gooch R., 1995, in Shaw R. A., Payne H. E., Hayes J. J. E., eds, ASP Conf. Ser. Vol. 77, Space and the Spaceball. Astron. Soc. Pac., San Francisco, p. 144

Haberl, F. and Pietsch, W., 1999, A\&AS, 139, 277

Hughes A., Staveley-Smith L., Kim S., Wolleben M., Filipović M., 2007, MNRAS, 382, 543

Maggi, P., Haberl, F., Kavanagh, P. J., Points, S. D., Dickel, J., Bozzetto, L. M., Sasaki, M., Chu, Y.-H., Gruendl, R. A., Filipović, M. D. and Pietsch, W., 2014, A\&A, 561, 76

Manchester, R. N., Damico, N. and Tuohy, I. R., 1985, Mon. Not. R. Astron. Soc., 212, 975

Mathewson, D. S., Ford, V. L., Dopita, M. A., Tuohy, I. R., Long, K. S., Helfand, D. J. 1983, ApJS, 51, 345

Mauch T., Murphy T., Buttery H. J., Curran J., Hunstead R. W., Piestrzynski B., Ropbertson J. G., Sadler E. M., 2008, VizieR Online Data Catalog, 8081, 0

Mills B. Y., Turtle A. J., Little A. G., Durdin J. M., 1984, Aust. J. Phys., 37, 321

Payne, J. L., White, G. L. and Filipović, M. D., 2008, MNRAS, 383, 1175
Pellegrini, E. W., Oey, M. S., Winkler, P. F., Points, S. D., Smith, R. C., Jaskot, A. E. and Zastrow, J., 2012, ApJ, 755,40

Rolfs, K., Wilson, T.: 2003: "Tools of Radio Astronomy 4ed.", Springer, Berlin.

Sault R. J., Wieringa M. H. 1994, A\&AS, 108, 585

Sault R. J., Teuben P. J., Wright M. C. H., 1995, in Shaw R. A., Payne H. E., Hayes J. J. E., eds, ASP Conf. Ser. Vol. 77, A Retrospective View of MIRIAD. p. 433

Seok, J. Y., Koo, B.-C., Onaka, T., Ita, Y., Lee, H.-G., Lee, J.-J., Moon, D.-S., Sakon, I., Kaneda, H., Lee, H. M., Lee, M. G. and Kim, S. E., 2008, PASJ, 60, 453

Urošević, D., Pannuti, T. G., Duric, N. and Theodorou, A., 2005, A\&A, 435, 437

Williams, R. M., Chu, Y.-H., Dickel, J. R., Petre, R., Smith, R. C. and Tavarez, M., 1999, ApJS, 123, 467

This manuscript was prepared with the AAS IATEX macros v5.2. 